\title{
Successive Complementary Expansion Method for Solving Troesch's Problem as a Singular Perturbation Problem
}

\author{
Süleyman Cengizci ${ }^{1}$ and Aytekin Eryılmaz ${ }^{2}$ \\ ${ }^{1}$ Insitute of Applied Mathematics, Middle East Technical University, 06800 Ankara, Turkey \\ ${ }^{2}$ Department of Mathematics, Nevsehir Haci Bektas Veli University, 50300 Nevsehir, Turkey \\ Correspondence should be addressed to Aytekin Eryılmaz; eryilmazaytekin@gmail.com
}

Received 8 May 2015; Accepted 4 October 2015

Academic Editor: Bhabani S. Dandapat

Copyright (c) 2015 S. Cengizci and A. Eryılmaz. This is an open access article distributed under the Creative Commons Attribution License, which permits unrestricted use, distribution, and reproduction in any medium, provided the original work is properly cited.

A simple and efficient method that is called Successive Complementary Expansion Method (SCEM) is applied for approximation to an unstable two-point boundary value problem which is known as Troesch's problem. In this approach, Troesch's problem is considered as a singular perturbation problem. We convert the hyperbolic-type nonlinearity into a polynomial-type nonlinearity using an appropriate transformation, and then we use a basic zoom transformation for the boundary layer and finally obtain a nonlinear ordinary differential equation that contains SCEM complementary approximation. We see that SCEM gives highly accurate approximations to the solution of Troesch's problem for various parameter values. Moreover, the results are compared with Adomian Decomposition Method (ADM) and Homotopy Perturbation Method (HPM) by using tables.

\section{Introduction}

Troesch's highly sensitive problem arises from a system of a nonlinear ordinary differential equations which occur in the investigation of the confinement of a plasma column by radiation pressure [1]. It also arises in the theory of gas porous electrodes $[2,3]$. The problem is defined by

$$
y^{\prime \prime}=\lambda \sinh (\lambda y), \quad 0 \leq x \leq 1,
$$

with the boundary conditions

$$
\begin{aligned}
& y(0)=0, \\
& y(1)=1,
\end{aligned}
$$

where $y=y(x)$ and $\lambda>0$. Roberts and Shipman [4] have shown that the closed form solution to problem (1) with the boundary conditions (2) in terms of the Jacobi elliptic function $\operatorname{sc}(n \mid m)$ is as follows:

$$
y(x)=\frac{2}{\lambda} \sinh ^{-1}\left[\frac{y^{\prime}(0)}{2} \mathrm{sc}\left(\lambda x \mid 1-\frac{1}{4}\left(y^{\prime}(0)\right)^{2}\right)\right],
$$

where $y^{\prime}(0)=2 \sqrt{1-m}$. The constant $m$ satisfies the transcendental equation

$$
\operatorname{sc}(\lambda \mid m)=\frac{\sinh (\lambda / 2)}{\sqrt{1-m}},
$$

where $\operatorname{sc}(\lambda \mid m)$ is the Jacobi function defined by $\operatorname{sc}(\lambda \mid$ $m)=\sin \phi / \cos \phi=\tan \phi$. Here $\phi, \lambda$, and $m$ are related by the integral

$$
\lambda=\int_{0}^{\phi} \frac{d \theta}{\sqrt{1-m \sin ^{2} \theta}} .
$$

It has been shown in $[4,5]$ that $y(x)$ has a singularity approximately located at

$$
x_{s} \approx \frac{1}{2 \lambda} \ln \left(\frac{16}{1-m}\right)=\frac{1}{\lambda} \ln \left(\frac{8}{y^{\prime}(0)}\right) .
$$

This singularity makes the problem very difficult to solve for large $\lambda$ values. 
The first explanation of Troesch's problem was given and solved by Weibel [1]. Because of its physical significance, Troesch's problem has always been attractive to scientists and various methods have been implemented to solve it such as Monte Carlo method [6], Modified Newton Method [7, 8], Transformation Groups Method [9], Invariant Embedded Method [10], Shooting Method [5], Inverse Shooting Method [11], The quasilinearization Method [12, 13], and Matched Asymptotic Expansions Method [14]. Recently, approximate solution techniques such as Adomian Decomposition Method [15, 16], Laplace transform and a modified decomposition technique [16], Variational Iteration Method [17, 18], Homotopy Perturbation Method [19], Differential Transform Method [20], B-spline collocation approach [21], The SincGalerkin Method [22], Wavelet Analysis Method [23], SincCollocation Method [24], and Jacobi Collocation Method [25] have been successfully applied to Troesch's problem by various researchers. In [26-29] more detailed information and references on Troesch's problem may also be seen.

In this paper, we consider Troesch's problem as a singular perturbation problem. By using appropriate transformations, we apply Successive Complementary Expansion Method (SCEM) to it. The results obtained by SCEM are compared with HPM and ADM and the analytic solutions.

\section{The Successive Complementary Expansion Method}

In this section, we first give a short overview of asymptotic approximations. One can consult [30] for some definitions on the asymptotic approximations and singular perturbations in more detail.

SCEM was first introduced by Mauss and Cousteix (see [30-34]). It has always been applied to partial differential equations except for some introductory examples in [30]. SCEM is based on the knowledge of generalized asymptotic expansions and the Method of Matched Asymptotic Expansions (MMAE). At first, a structure of the uniformly valid approximation (UVA) must be assumed and then the method to construct the UVA is deduced [32, 33]. In this method, any matching procedure is required in contrast to MMAE. Furthermore, the boundary conditions are satisfied exactly but not asymptotically.

Let us consider a sequence of real numbers $\{f(n)\}_{n=1}^{\infty}$. We are familiar with the limit notation $\lim _{n \rightarrow \infty} f(n)$. If this limit exists we can denote it as $\lim _{n \rightarrow \infty} f(n)=f \in \mathbb{R}$. We can give the following definition: given any $\varepsilon>0$ there exists a number $n_{0}(\varepsilon)$ such that $|f(n)-f|<\varepsilon$ for any $n>n_{0}(\varepsilon)$. This definition contains information about the behaviour of $f(n)$ as $n \rightarrow \infty$ but not about how $f(n)$ approaches $f$. In order to describe the behaviour of sequences in a more precise way, we need some other definitions, Bachmann-Landau notations. Let us consider two continuous functions of real numbers that depend on a small parameter $\varepsilon f(\varepsilon)=O(g(\varepsilon))$ for $\varepsilon \rightarrow 0$ if there exists positive constants $C$ and $\varepsilon_{0}$ such that, in $\left(0, \varepsilon_{0}\right]$, $|f(\varepsilon)| \leqslant C|g(\varepsilon)|$ for $\varepsilon \rightarrow 0$. $f(\varepsilon)=o(g(\varepsilon))$ for $\varepsilon \rightarrow 0$ if $\lim _{\varepsilon \rightarrow 0}(f(\varepsilon) / g(\varepsilon))=0 . f(\varepsilon)=O_{S}(g(\varepsilon))$ if $f(\varepsilon)=O(g(\varepsilon))$ and $f(\varepsilon) \neq o(g(\varepsilon))$ as $\varepsilon \rightarrow 0$. Let $E$ be a set of real functions that depend on $\varepsilon$, strictly positive and continuous in $\left(0, \varepsilon_{0}\right]$, such that $\lim _{\varepsilon \rightarrow 0} \delta(\varepsilon)$ exists and if $\delta_{1}$ and $\delta_{2} \in E, \delta_{1} \delta_{2} \in$ $E$. A function that satisfies these conditions is called order function. Given two functions $\phi(x, \varepsilon)$ and $\phi_{a}(x, \varepsilon)$ defined in a domain $\Omega$, they are asymptotically identical to order $\delta(\varepsilon)$ if their difference is asymptotically smaller than $\delta(\varepsilon)$, where $\delta(\varepsilon)$ is an order function; that is,

$$
\phi(x, \varepsilon)-\phi_{a}(x, \varepsilon)=o(\delta(\varepsilon)),
$$

where $\varepsilon$ is small parameter arising from the physical problem under consideration. The function $\phi_{a}(x, \varepsilon)$ is named as asymptotic approximation of the function $\phi(x, \varepsilon)$. Asymptotic approximations in general form are defined by

$$
\phi_{a}(x, \varepsilon)=\sum_{i=1}^{n} \delta_{i}(\varepsilon) \varphi_{i}(x, \varepsilon)
$$

where the asymptotic sequence of order functions $\delta_{i}(\varepsilon)$ is an asymptotic sequence such that $\delta_{i+1}(\varepsilon)=o\left(\delta_{i}(\varepsilon)\right)$, as $\varepsilon \rightarrow 0$. In these conditions approximation (8) is named as generalized asymptotic expansion. If expansion (8) is written in the form of

$$
\phi_{a}(x, \varepsilon)=E_{0} \phi=\sum_{i=1}^{n} \delta_{i}^{(0)}(\varepsilon) \varphi_{i}^{(0)}(x),
$$

then it is called regular asymptotic expansion, where the special operator $E_{0}$ is outer expansion operator at a given order $\delta(\varepsilon)$. Thus $\phi-E_{0} \phi=o(\delta(\varepsilon))$. For more detailed information about the asymptotic approximations, $[10,11$, $13,19]$ can be studied. Interesting cases occur when the function is not regular in $\Omega$ so (8) or (9) is valid only in a restricted region $\Omega_{0} \in \Omega$ called the outer region. We are faced with a singular perturbation problem and we must introduce boundary layer domains. We introduce an inner domain which can be formally denoted as $\Omega_{1}=\Omega_{0}-\Omega$ and located near the point $x=1$ (for Troesch's problem). The boundary layer variable is $\bar{x}=(x-1) / \xi(\varepsilon)$, with $\xi(\varepsilon)$ being the order of thickness of this boundary layer. If a regular expansion can be constructed in $\Omega_{1}$, we can write down

$$
\phi_{a}(x, \varepsilon)=E_{1} \phi=\sum_{i=0}^{n} \delta_{i}^{(1)}(\varepsilon) \varphi_{i}^{(1)}(\bar{x}),
$$

where the inner expansion operator $E_{1}$ is defined in $\Omega_{1}$ at the same order $\delta(\varepsilon)$ as the outer expansion operator $E_{0}$; thus, $\phi-$ $E_{1} \phi=o(\delta(\varepsilon))$ and

$$
\phi_{a}=E_{0} \phi+E_{1} \phi-E_{1} E_{0} \phi
$$

is clearly uniformly valid approximation (UVA) $[35,36]$. In the Method of Matched Asymptotic Expansions (MMAE) [14], two distinct solutions of the singular perturbation problem are found for two distinct regions (inner and outer) and then to obtain uniformly valid approximation over the whole domain the solutions are matched using limit process

$$
\lim _{x \rightarrow 1} y_{0}(x)=\lim _{\bar{x} \rightarrow-\infty} Y(\bar{x})
$$


where $Y(\bar{x})$ is boundary layer approximation. Finally uniformly valid approximation is obtained by simply adding the inner and outer solutions and subtracting the common limit. That is, using the procedure

$$
y \approx y_{0}(x)+Y_{0}(\bar{x})-Y_{0}(-\infty),
$$

or equivalently

$$
y \approx y_{0}(x)+Y_{0}(\bar{x})-y_{0}\left(1^{-}\right),
$$

one can reach the composite solution which is uniformly valid over the whole domain. The uniformly valid SCEM approximation is in the regular form

$$
y_{n}^{\text {scem }}(x, \bar{x}, \epsilon)=\sum_{i=1}^{n} \delta_{i}(\varepsilon)\left[y_{i}(x)+\Psi_{i}(\bar{x})\right],
$$

where $\delta_{i}$ is an asymptotic sequence and $\Psi_{i}(\bar{x})$ are the complementary functions that depend on $\bar{x}$. Functions $y_{i}(x)$ are the outer approximation functions that have been found by MMAE and they only depend on $x$, not also $\varepsilon$. If the functions $y_{i}(x)$ and $\Psi_{i}(\bar{x})$ depend also on $\varepsilon$, the uniformly valid SCEM approximation is called generalized SCEM approximation that is in the following form [30, 31]:

$$
y_{n g}^{\text {scem }}(x, \bar{x}, \epsilon)=\sum_{i=1}^{n} \delta_{i}(\varepsilon)\left[y_{i}(x, \varepsilon)+\Psi_{i}(\bar{x}, \varepsilon)\right] .
$$

For the sake of simplicity, we adopt just one-term approximation in this study. That is, we look for an approximation in the form of

$$
y_{0}^{\text {scem }}(x, \bar{x}, \varepsilon)=y_{0}(x, \varepsilon)+\Psi_{0}(\bar{x}, \varepsilon) .
$$

To improve the accuracy of approximation, (17) can be iterated using (16). It means that successive complementary terms will be added to the approximation.

\section{Application of SCEM to Troesch's Problem}

In this section, we solve Troesch's problems for different values of the parameter $\lambda$ using the computer algebra system Matlab and make a comparison between our results and those ones reported in the literature to confirm the efficiency and accuracy of our method. In first step, let us turn Troesch's problem into singular perturbation problem as follows:

$$
y^{\prime \prime}=\lambda \sinh (\lambda y) \text {. }
$$

Letting $\lambda=1 / \varepsilon$ in (18), we obtain

$$
\begin{aligned}
y^{\prime \prime} & =\frac{1}{\varepsilon} \sinh \left(\frac{y}{\varepsilon}\right), \\
\varepsilon y^{\prime \prime}-\sinh \left(\frac{y}{\varepsilon}\right) & =0 .
\end{aligned}
$$

Now, in order to avoid overflow or excessive error growth during numerical integration, removing the hyperbolic-type nonlinearity using the variable transformation, which was originally proposed in [36], $y(x)=4 \varepsilon \tanh ^{-1}(u(x))$ or equivalently $u(x)=\tanh (y(x) / 4 \varepsilon)$, we get

$$
\begin{aligned}
y^{\prime} & =\frac{4 \varepsilon}{\left(1-u^{2}\right)} u^{\prime} \\
y^{\prime \prime} & =\frac{4 \varepsilon}{\left(1-u^{2}\right)} u^{\prime \prime}+\frac{8 \varepsilon u}{\left(1-u^{2}\right)^{2}}\left(u^{\prime}\right)^{2}
\end{aligned}
$$

or

$$
\left(1-u^{2}\right) u^{\prime \prime}+2 u\left(u^{\prime}\right)^{2}=\frac{u\left(1+u^{2}\right)}{\varepsilon^{2}}
$$

and finally Troesch's problem has the form

$$
\varepsilon^{2} u^{\prime \prime}+\frac{2 \varepsilon^{2} u\left(u^{\prime}\right)^{2}}{\left(1-u^{2}\right)}-\frac{u\left(1+u^{2}\right)}{\left(1-u^{2}\right)}=0
$$

with the boundary conditions

$$
\begin{aligned}
& u(0)=0, \\
& u(1)=\tanh \left(\frac{1}{4 \varepsilon}\right) .
\end{aligned}
$$

We reach polynomial-type nonlinearity instead of hyperbolic-type nonlinearity. The problem has the outer solution (near the point $x=0$ ) $y_{0}(x)=0$ (or equivalently $\left.u_{0}(x)=0\right)$. So the complementary solution directly produces approximation to Troesch's problem. If we substitute the inner variable $\bar{x}=(x-1) / \varepsilon$ into (22) and boundary conditions (23), using the chain rule, we reach

$$
\Psi_{0}^{\prime \prime}+\frac{2 \Psi_{0}\left(\Psi_{0}^{\prime}\right)^{2}-\Psi_{0}\left(1+\Psi_{0}^{2}\right)}{1-\Psi_{0}^{2}}=0
$$

and equivalently

$$
\Psi_{0}^{\prime \prime}+\frac{\Psi_{0}\left(2\left(\Psi_{0}^{\prime}\right)^{2}-\Psi_{0}^{2}-1\right)}{1-\Psi_{0}^{2}}=0
$$

with the boundary conditions

$$
\begin{aligned}
\Psi_{0}\left(\frac{-1}{\varepsilon}\right) & =0, \\
\Psi_{0}(0) & =\tanh \left(\frac{1}{4 \varepsilon}\right),
\end{aligned}
$$

where $\Psi_{0}$ is the complementary function. Here, the complementary function of SCEM, $\Psi_{0}$, is calculated numerically by Matlab bvp $4 c$ routine. Results which are obtained using SCEM are presented in the following section. Applying SCEM, we reach really highly accurate approximations to Troesch's problem. 
TABLE 1: Solution of Troesch's problem for $\varepsilon=2$.

\begin{tabular}{lccccc}
\hline$x$ & Exact solution & SCEM & ADM [13] & HPM [16] & Abs. err. in SCEM \\
\hline 0.1 & 0.09594435 & 0.09594435 & 0.09593835 & 0.09593956 & $3.13441692 e(-9)$ \\
0.2 & 0.19212874 & 0.19212875 & 0.19211805 & 0.19211932 & $7.32982755 e(-9)$ \\
0.3 & 0.28879440 & 0.28879441 & 0.28878032 & 0.28878069 & $1.30811447 e(-8)$ \\
0.4 & 0.38618485 & 0.38618487 & 0.38616870 & 0.38616754 & $1.98750830 e(-8)$ \\
0.5 & 0.48454716 & 0.48454719 & 0.48453029 & 0.48452741 & $2.60076459 e(-8)$ \\
0.6 & 0.58413325 & 0.58413328 & 0.58411697 & 0.58411278 & $2.88212714 e(-8)$ \\
0.7 & 0.68520115 & 0.68520118 & 0.68518684 & 0.68518224 & $2.55610896 e(-8)$ \\
0.8 & 0.78801652 & 0.78801654 & 0.78800556 & 0.78800183 & $1.51078886 e(-8)$ \\
0.9 & 0.89285422 & 0.89285422 & 0.89284802 & 0.89284621 & $9.280242130 e(-10)$ \\
\hline
\end{tabular}

TABLE 2: Solution of Troesch's problem for $\varepsilon=1$.

\begin{tabular}{lccccc}
\hline$x$ & Exact solution & SCEM & ADM [13] & HPM [16] & Abs. err. in SCEM \\
\hline 0.1 & 0.08466141 & 0.08466143 & 0.08424876 & 0.08438170 & $2.18232920 e(-8)$ \\
0.2 & 0.17017167 & 0.17017172 & 0.16943070 & 0.16962076 & $5.16987398 e(-8)$ \\
0.3 & 0.25739438 & 0.25739447 & 0.25641450 & 0.25659292 & $8.76146832 e(-8)$ \\
0.4 & 0.34722348 & 0.34722359 & 0.34608572 & 0.34621073 & $1.09822865 e(-7)$ \\
0.5 & 0.44060059 & 0.44060067 & 0.43940198 & 0.43944227 & $7.88214280 e(-8)$ \\
0.6 & 0.53853523 & 0.53853518 & 0.53736570 & 0.53733006 & $5.56789394 e(-8)$ \\
0.7 & 0.64212944 & 0.64212911 & 0.64108380 & 0.64101046 & $3.25396753 e(-7)$ \\
0.8 & 0.75260880 & 0.75260812 & 0.75178800 & 0.75173354 & $6.76312419 e(-7)$ \\
0.9 & 0.87136294 & 0.87136211 & 0.87090870 & 0.87088353 & $8.29947601 e(-7)$ \\
\hline
\end{tabular}

TABLE 3: Absolute error of SCEM approximation for $\varepsilon=0.1$.

\begin{tabular}{lccc}
\hline$x$ & Exact solution & SCEM & Abs. err. in SCEM \\
\hline 0.10 & 0.000004211 & 0.000042111 & $3.79006047 e(-5)$ \\
0.20 & 0.000129963 & 0.000129964 & $1.57300000 e(-10)$ \\
0.30 & 0.000358977 & 0.000358978 & $4.20000000 e(-10)$ \\
0.40 & 0.000977901 & 0.000977902 & $1.08799999 e(-9)$ \\
0.50 & 0.002659017 & 0.002659019 & $2.60240000 e(-9)$ \\
0.60 & 0.007228924 & 0.007228929 & $4.58729999 e(-9)$ \\
0.70 & 0.019664060 & 0.019664057 & $2.41529999 e(-9)$ \\
0.80 & 0.053730329 & 0.053730314 & $1.46944000 e(-8)$ \\
0.90 & 0.152114078 & 0.152114033 & $4.55675999 e(-8)$ \\
0.95 & 0.276267734 & 0.276267643 & $9.12888999 e(-8)$ \\
0.99 & 0.574076498 & 0.574076080 & $4.17938999 e(-7)$ \\
\hline
\end{tabular}

\section{Conclusions}

In this study, an efficient method called Successive Complementary Expansion Method (SCEM) is used to solve the nonlinear two-point boundary value problem with application to Troesch's equation. SCEM is an easy-applicable and efficient method. It does not require any matching procedure in contrast to MMAE. Moreover, the boundary conditions are satisfied exactly, not asymptotically. In Tables 1-3, the

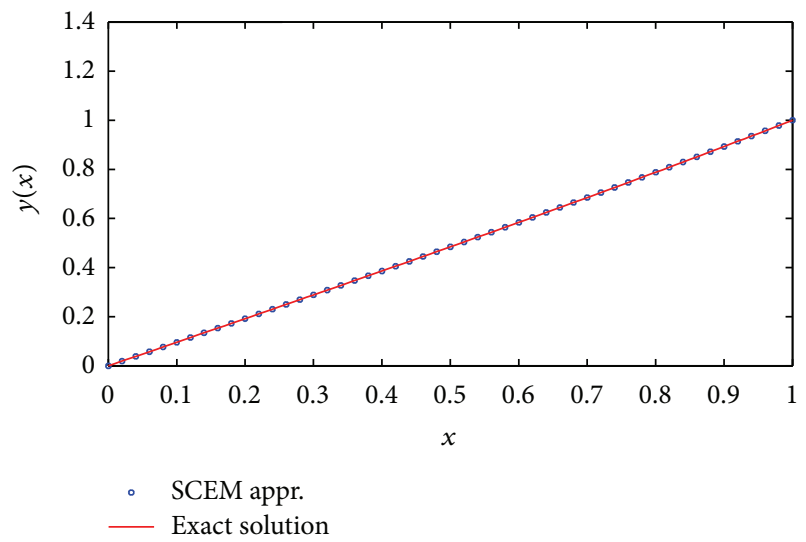

FIGURE 1: Exact solution and SCEM approximation of Troesch's problem for $\varepsilon=2$.

absolute errors in solutions obtained by the presented method for $\varepsilon=2, \varepsilon=1$, and $\varepsilon=0.1$, respectively, are shown. In Figures 1-5, we compare exact and SCEM solutions. The results obtained here were compared with the exact solution, ADM [15], and HPM [19]. It is shown that the method is computationally attractive and the presented method is much 


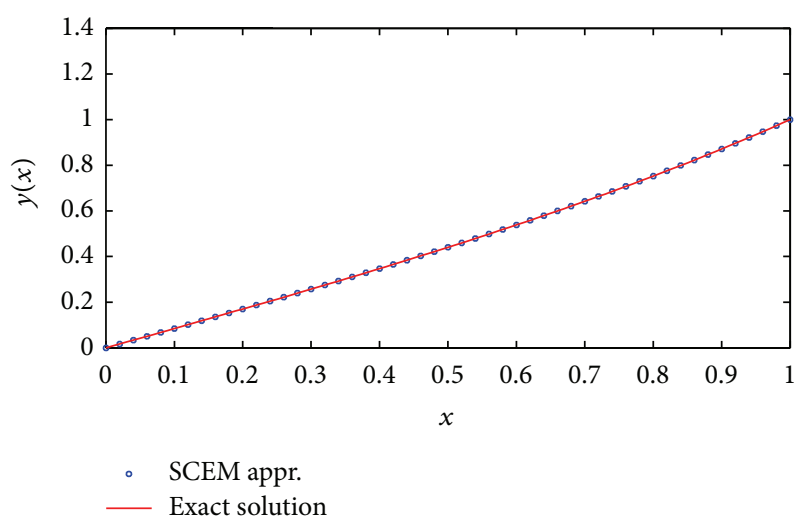

FIGURE 2: Exact solution and SCEM approximation of Troesch's problem for $\varepsilon=1$.

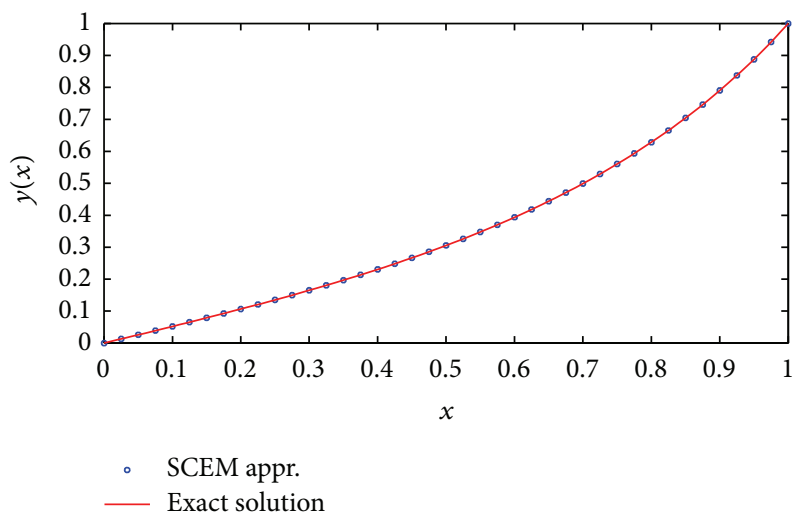

FIGURE 3: Exact solution and SCEM approximation of Troesch's problem for $\varepsilon=0.5$.

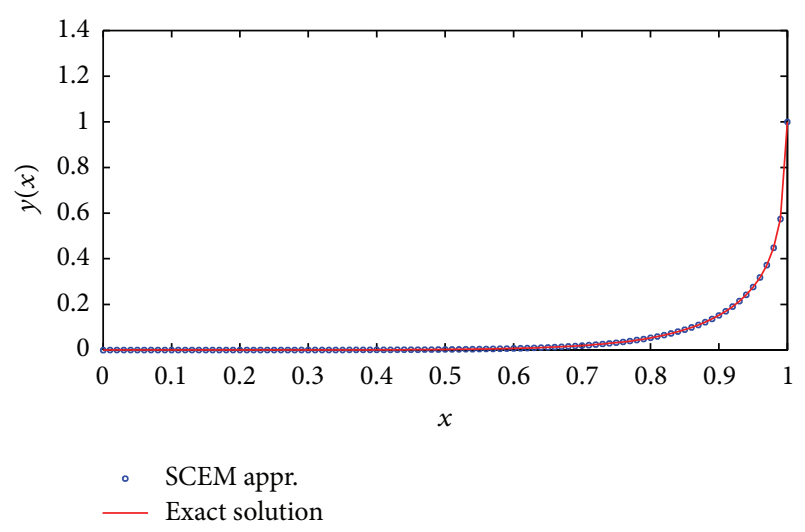

FIGURE 4: Exact solution and SCEM approximation of Troesch's problem for $\varepsilon=0.1$.

better than other reported ones in the literature in the sense of accuracy and efficiency.

\section{Conflict of Interests}

The authors declare that they have no conflict of interests regarding the publication of this paper.

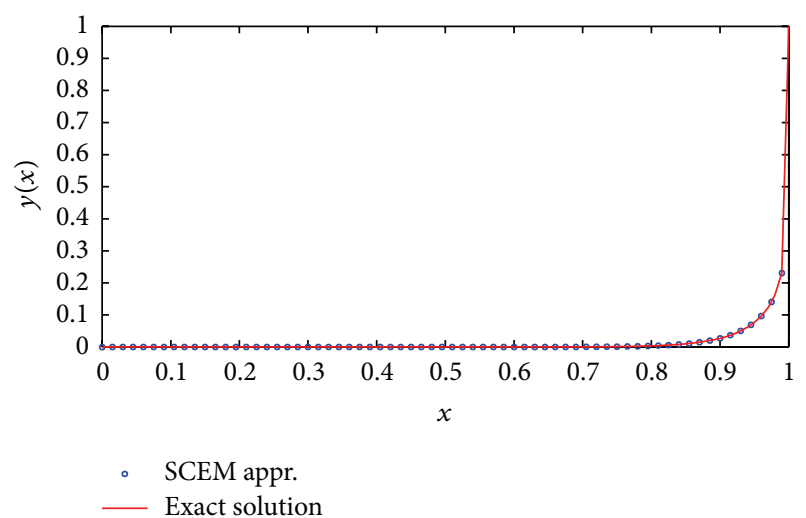

FIGURE 5: Exact solution and SCEM approximation of Troesch's problem for $\varepsilon=0.05$.

\section{References}

[1] E. S. Weibel, "On the confinement of a plasma by magnetostatic fields," Physics of Fluids, vol. 2, no. 1, pp. 52-56, 1959.

[2] V. S. Markin, A. A. Chernenko, Y. A. Chizmadehev, and Y. G. Chirkov, "Aspects of the theory of gas porous electrodes", in Fuel Cells: Their Electrochemical Kinetics, V. S. Bagotskii and Y. B. Vasilev, Eds., pp. 21-33, Consultants Bureau, New York, NY, USA, 1966.

[3] D. Gidaspow and B. S. Baker, "A model for discharge of storage batteries," Journal of the Electrochemical Society, vol. 120, no. 8, pp. 1005-1010, 1973.

[4] S. M. Roberts and J. S. Shipman, "On the closed form solution of Troesch's problem," Journal of Computational Physics, vol. 21, no. 3, pp. 291-304, 1976.

[5] B. A. Troesch, "A simple approach to a sensitive two-point boundary value problem," Journal of Computational Physics, vol. 21, no. 3, pp. 279-290, 1976.

[6] T. Tsuda, K. Ichida, and T. Kiyono, "Monte Carlo pathintegral calculations for two-point boundary-value problems," Numerische Mathematik, vol. 10, no. 2, pp. 110-116, 1967.

[7] D. J. Jones, "Solution of Troesch's, and other, two point boundary value problems by shooting techniques," Journal of Computational Physics, vol. 12, no. 3, pp. 429-434, 1973.

[8] M. Kubiček and V. Hlaváček, "Solution of Troesch's two-point boundary value problem by shooting technique," Journal of Computational Physics, vol. 17, no. 1, pp. 95-101, 1975.

[9] J. P. Chiou and T. Y. Na, "On the solution of Troesch's nonlinear two-point boundary value problem using an initial value method," Journal of Computational Physics, vol. 19, no. 3, pp. 311316, 1975.

[10] M. R. Scott, "On the conversion of boundary-value problems into stable initial-value problems via several invariant imbedding algorithms," in Numerical Solutions of Boundary-Value Problems for Ordinary Differential Equations, A. K. Aziz, Ed., pp. 89-146, Academic Press, New York, NY, USA, 1975.

[11] J. A. Snyman, "Continuous and discontinuous numerical solutions to the Troesch problem," Journal of Computational and Applied Mathematics, vol. 5, no. 3, pp. 171-175, 1979.

[12] A. Miele, A. K. Aggarwal, and J. L. Tietze, "Solution of two-point boundary-value problems with Jacobian matrix characterized by large positive eigenvalues," Journal of Computational Physics, vol. 15, no. 2, pp. 117-133, 1974. 
[13] V. Vemuri and A. Raefsky, "On a method of solving sensitive boundary value problems," Journal of the Franklin Institute, vol. 307, no. 4, pp. 217-243, 1979.

[14] R. C. Y. Chin, "On the matched asymptotic solution of the Troesch problem," Journal of Computational and Applied Mathematics, vol. 7, no. 3, pp. 181-185, 1981.

[15] E. Deeba, S. A. Khuri, and S. Xie, "An algorithm for solving boundary value problems," Journal of Computational Physics, vol. 159, no. 2, pp. 125-138, 2000.

[16] S. A. Khuri, "A numerical algorithm for solving Troesch's problem," International Journal of Computer Mathematics, vol. 80, no. 4, pp. 493-498, 2003.

[17] S. Momani, S. Abuasad, and Z. Odibat, "Variational iteration method for solving nonlinear boundary value problems," Applied Mathematics and Computation, vol. 183, no. 2, pp. 13511358, 2006.

[18] S.-H. Chang, "A variational iteration method for solving Troesch's Problem," Journal of Computational and Applied Mathematics, vol. 234, no. 10, pp. 3043-3047, 2010.

[19] X. Feng, L. Mei, and G. He, "An efficient algorithm for solving Troesch's problem," Applied Mathematics and Computation, vol. 189, no. 1, pp. 500-507, 2007.

[20] S.-H. Chang and I.-L. Chang, "A new algorithm for calculating one-dimensional differential transform of nonlinear functions," Applied Mathematics and Computation, vol. 195, no. 2, pp. 799808, 2008.

[21] S. A. Khuri and A. Sayfy, "Troesch's problem: a B-spline collocation approach," Mathematical and Computer Modelling, vol. 54, no. 9-10, pp. 1907-1918, 2011.

[22] M. Zarebnia and M. Sajjadian, "The sinc-galerkin method for solving Troesch's problem," Mathematical and Computer Modelling, vol. 56, no. 9-10, pp. 218-228, 2012.

[23] A. K. Nasab, Z. P. Atabakan, and A. Kılıçman, "An efficient approach for solving nonlinear Troesch's and Bratu's problems by wavelet analysis method," Mathematical Problems in Engineering, vol. 2013, Article ID 825817, 10 pages, 2013.

[24] M. El-Gamel, "Numerical solution of Troesch's problem by sinccollocation method," Applied Mathematics, vol. 4, no. 4, pp. 707$712,2013$.

[25] E. H. Doha, D. Baleanu, A. H. Bhrawi, and R. M. Hafez, "A Jacobi collocation method for Troesch's problem in plasma physics," Proceedings of the Romanian Academy, Series A, vol. 15, no. 2, pp. 130-138, 2014.

[26] L. Bougoffa and M. A. Al-khadhi, "New explicit solutions for Troeschs boundary value problem," Applied Mathematics \& Information Sciences, vol. 3, no. 1, p. 8996, 2009.

[27] S. H. Mirmoradi, I. Hosseinpour, S. Ghanbarpour, and A. Barari, "Application of an approximate analytical method to nonlinear Troesch's problem," Applied Mathematical Sciences, vol. 3, no. 29-32, pp. 1579-1585, 2009.

[28] H. N. Hassan and M. A. El-Tawil, "An efficient analytic approach for solving two-point nonlinear boundary value problems by homotopy analysis method," Mathematical Methods in the Applied Sciences, vol. 34, no. 8, pp. 977-989, 2011.

[29] S. T. Mohyud-Din, "Solution of Troesch's problem using He's polynomials," Revista de la Unión Matemática Argentina, vol. 52, no. 1, pp. 143-148, 2011.

[30] J. Cousteix and J. Mauss, Asymptotic Analysis and Boundary Layers, Scientific Computation, Springer, Berlin, Germany, 2007.
[31] J. Cousteix and J. Mauss, "Approximations of the Navier-Stokes equations for high Reynolds number flows past a solid wall," Journal of Computational and Applied Mathematics, vol. 166, no. 1, pp. 101-122, 2004.

[32] J. Mauss and J. Cousteix, "Uniformly valid approximation for singular perturbation problems and matching principle," Comptes Rendus Mécanique, vol. 330, no. 10, pp. 697-702, 2002.

[33] J. Cousteix and J. Mauss, "Interactive boundary layers in turbulent flow," Comptes Rendus Mécanique, vol. 335, no. 9-10, pp. 590-605, 2007.

[34] J. Mauss, “On matching principles," in Asymptotic Analysis, vol. 711 of Lecture Notes in Mathematics, pp. 1-8, Springer, Berlin, Germany, 1979.

[35] P. Cathalifaud, J. Mauss, and J. Cousteix, "Nonlinear aspects of high Reynolds number channel flows," European Journal of Mechanics-B/Fluids, vol. 29, no. 4, pp. 295-304, 2010.

[36] P. A. Lagerstrom, Matched Asymptotic Expansions: Ideas and Techniques, vol. 76 of Applied Mathematical Sciences, Springer, New York, NY, USA, 1988. 


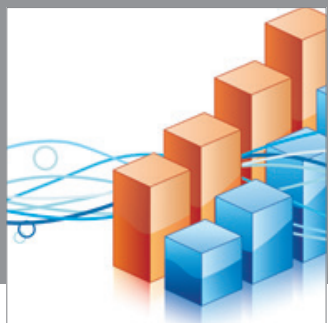

Advances in

Operations Research

mansans

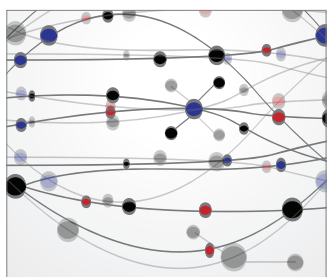

The Scientific World Journal
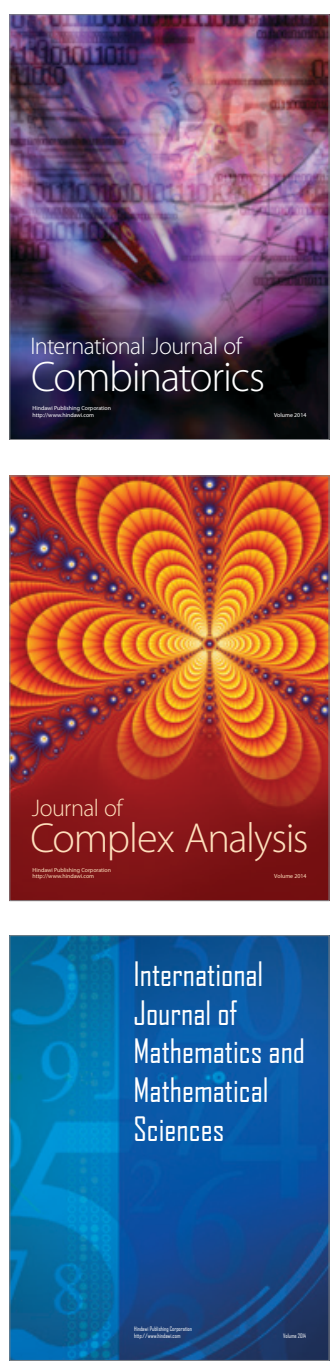
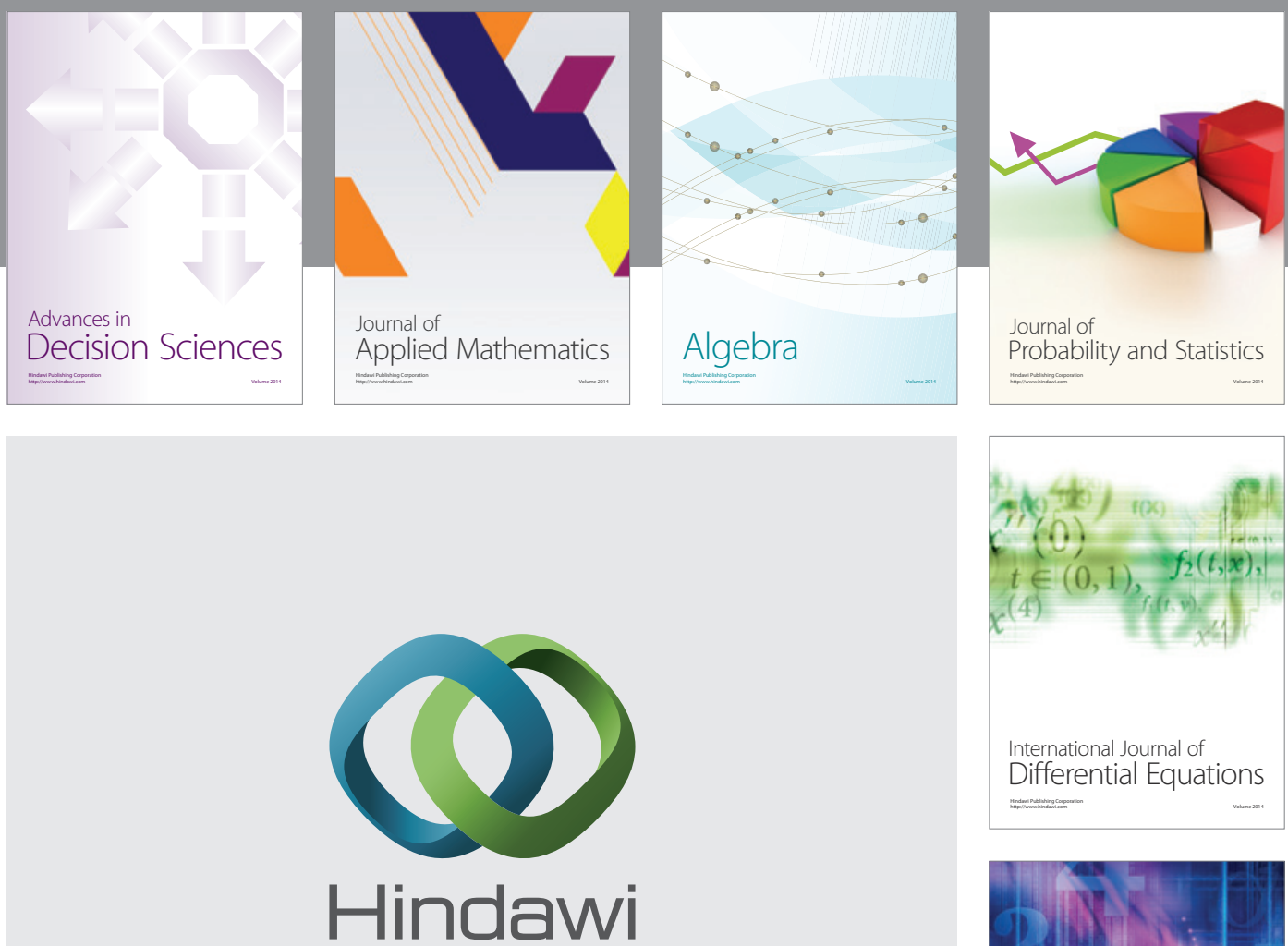

Submit your manuscripts at http://www.hindawi.com
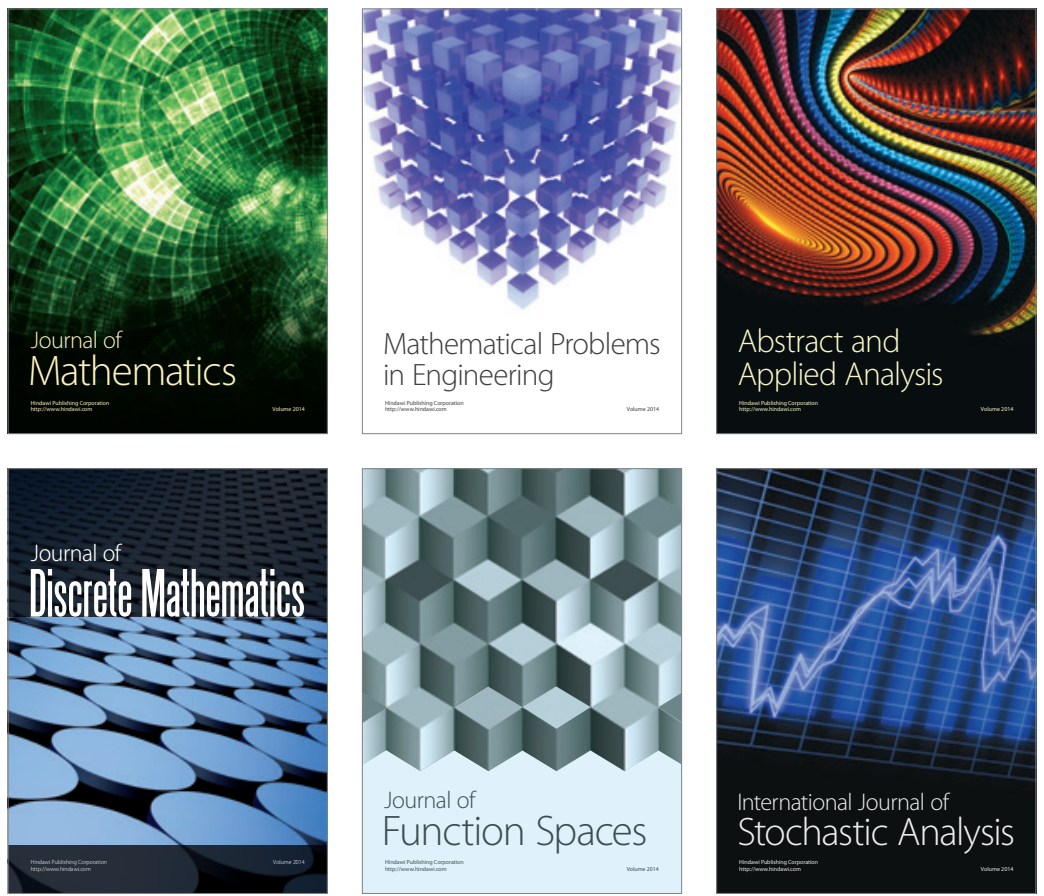

Journal of

Function Spaces

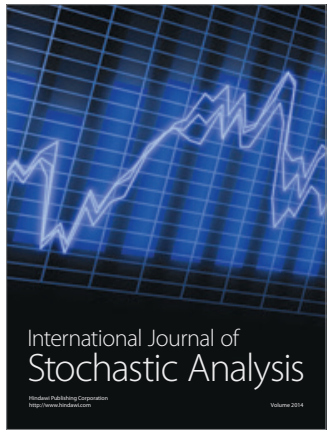

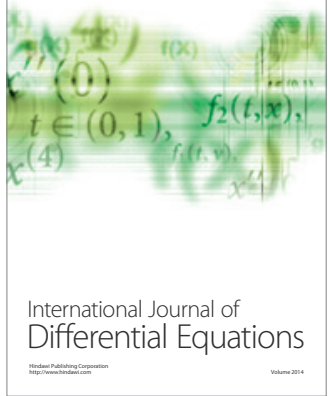
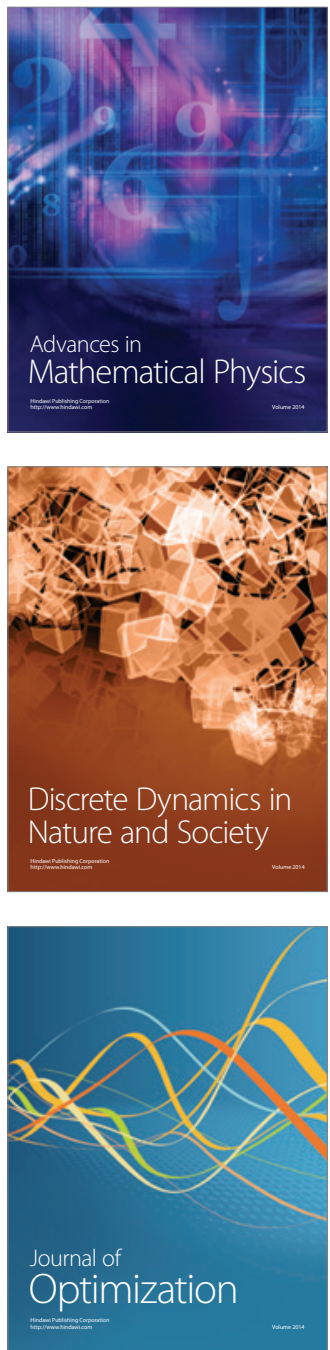